\title{
Scaffold 877
}

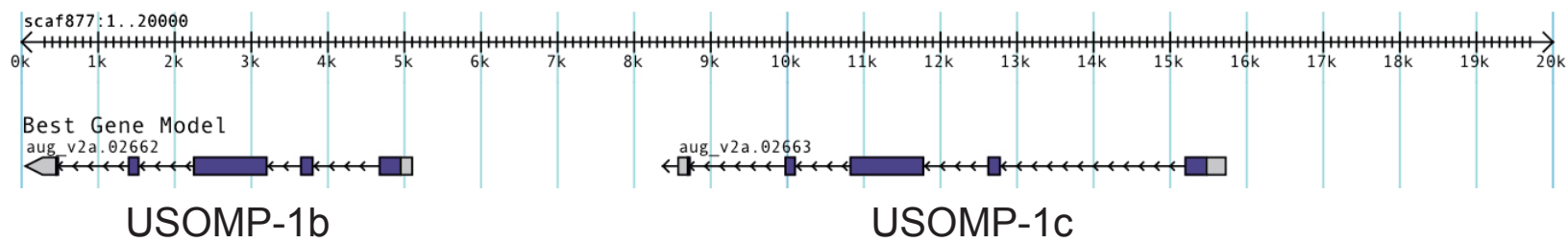

Scaffold 2257

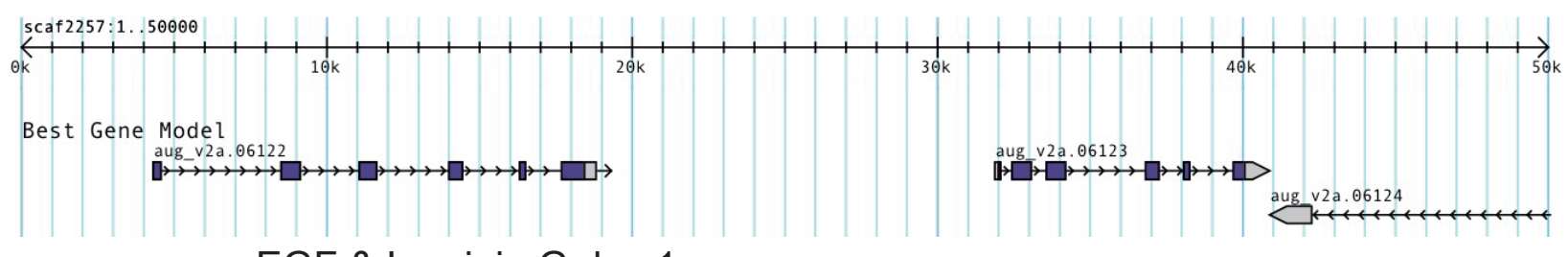

EGF \& Laminin G dcp 1

EGF \& Laminin G dcp 2

Scaffold 6350

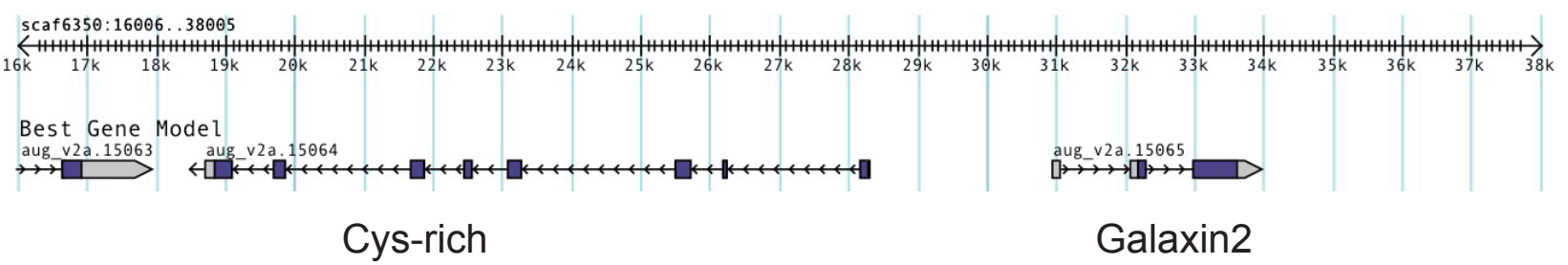

S23 Fig. Tandem arrangement of SOMP genes in the A. digitifera genome scaffold. 